\title{
Correction to: Isolated Human and Livestock Echinococcus granulosus Genotypes Using Real-Time PCR of cox1 Gene in Northeast Iran
}

\author{
Mohammad-Ali Mohaghegh ${ }^{1} \cdot$ Hossein Yousofi-Darani ${ }^{1} \cdot$ Amir Hossein Jafarian $^{2} \cdot$ Seyed-Reza Mirbadie $^{3}$. \\ Majid Fasihi-Harandi ${ }^{4} \cdot$ Reza Ghavimi $^{5} \cdot$ Zahra Jabalameli $^{6} \cdot$ Mehdi Azami $^{7} \cdot$ Mohsen Mohammadi $^{8}$. \\ Seyed Hossein Hejazi ${ }^{9}$
}

Published online: 15 October 2019

๑) Witold Stefański Institute of Parasitology, Polish Academy of Sciences 2019

\section{Correction to: Acta Parasitologica https://doi.org/10.2478/s11686-019-00117-w}

Unfortunately, the affiliation of Majid Fasihi-Harandi needs to be edited. The correct affiliation is Research Center for Hydatid Disease in Iran, School of Medicine, Kerman University of Medical Sciences, Kerman, Iran.

Publisher's Note Springer Nature remains neutral with regard to jurisdictional claims in published maps and institutional affiliations.

The original article can be found online at https://doi.org/10.2478/ s11686-019-00117-w.

Seyed Hossein Hejazi

hejazi@med.mui.ac.ir

1 Department of Parasitology and Mycology, School of Medicine, Isfahan University of Medical Sciences, Isfahan, Iran

2 Department of Pathology, Faculty of Medicine, Mashhad University of Medical Sciences, Mashhad, Iran

3 School of Medicine, Shahroud University of Medical Sciences, Shahroud, Iran

4 Research Center for Hydatid Disease in Iran, School of Medicine, Kerman University of Medical Sciences, Kerman, Iran

5 Department of Pharmaceutical Biotechnology, School of Pharmacy, Isfahan University of Medical Sciences, Isfahan, Iran
6 Department of Laboratory Sciences, School of Paramedical Sciences, Torbat Heydariyeh University of Medical Sciences, Torbat Heydariyeh, Iran

7 Skin Diseases and Leishmaniasis Research Center, Isfahan University of Medical Sciences, Isfahan, Iran

8 The Persian Gulf Marine Biotechnology Research Center, Persian Gulf Biomedical Sciences Research Institute, Bushehr University of Medical Sciences, Bushehr, Iran

9 Department of Parasitology and Mycology, School of Medicine, Skin Diseases and Leishmaniasis Research Center, Isfahan University of Medical Sciences, Isfahan, Iran 